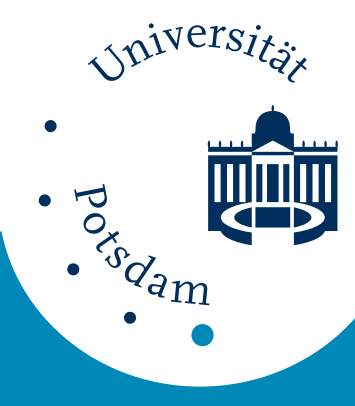

Universität Potsdam

Hans-Gerd Löhmannsröben, Toralf Beitz,

Robert Laudien, Rainer Schultze

\title{
Laser-based ion mobility spectrometry for sensing of aromatic compounds
}

first published in:

Remote Sensing in Atmosperic Pollution Monitoring and Control / Allen Chu, James Szykman (eds.). - S. 16 - 24. - (Proceedings of SPIE ; 5547) doi: $10.1117 / 12.559509$

Postprint published at the institutional repository of Potsdam University: In: Postprints der Universität Potsdam :

Mathematisch-Naturwissenschaftliche Reihe ; 3

http://opus.kobv.de/ubp/volltexte/2007/1189/

http://nbn-resolving.de/urn:nbn:de:kobv:517-opus-11892

Postprints der Universität Potsdam

Mathematisch-Naturwissenschaftliche Reihe ; 3 


\title{
Laser-based ion mobility spectrometry for sensing of aromatic compounds
}

\author{
H.-G. Löhmannsröben ${ }^{*}$, T. Beitz ${ }^{\mathrm{a}}$, R. Laudien ${ }^{\mathrm{a}}$, R. Schultze ${ }^{\mathrm{b}}$ \\ Institute of Chemistry, Physical Chemistry, University of Potsdam, Karl-Liebknecht-Str. \\ 24-25, D-14476 Potsdam-Golm, Germany \\ ${ }^{b}$ Optimare GmbH, Jadestr. 59, D-26382 Wilhelmshaven, Germany
}

\begin{abstract}
The drift time spectra of polycyclic aromatic hydrocarbons (PAH), alkylbenzenes and alkylphenylethers were recorded with a laser-based ion mobility (IM) spectrometer. The ion mobilities of all compounds were determined in helium as drift gas. This allows the calculation of the diffusion cross sections $\left(\Omega_{\text {calc }}\right)$ on the basis of the exact hard sphere scattering model (EHSSM) and their comparison with the experimentally determined diffusion cross sections $\left(\Omega_{\text {exp }}\right)$. These $\Omega_{\text {exp }} / \Omega_{\text {calc }}$-correlations are presented for molecules with a rigid structure like PAH and prove the reliability of the theoretical model and experimental method. The increase of the selectivity of IM spectrometry is demonstrated using resonance enhanced multiphoton ionisation (REMPI) at atmospheric pressure, realized by tuneable lasers. The REMPI spectra of nine alkylbenzenes and alkylphenylethers are investigated. On the basis of these spectra, the complete qualitative distinction of eight compounds in a mixture is shown. These experiments are extended to alkylbenzene isomer mixtures.
\end{abstract}

Keywords: Laser, REMPI, Ion mobility spectrometry, PAH

\section{INTRODUCTION}

IM spectrometry is based on the drift movement of molecular ions in a bath gas under the influence of an external electrical field and allows the determination of ion mobilities. The drift time profiles obtained are referred to as IM spectra. An important usage of IM spectrometry is its application for gas sensing in chemical analysis. IM spectrometry essentially works at atmospheric pressure (i.e., no vacuum technique is required) and does not need expensive detector units. In comparison to mass spectrometric methods, IM spectrometers are compact and less expensive. The IM technique is established for mobile, on-site investigations in process and environmental analysis. ${ }^{1-3}$ Analytical applications range from the detection of BTEX aromatic compounds over drug screening to the detection of explosives and chemical warfare agents. ${ }^{2,4}$ Commercially available IM spectrometers, offering high sensitivity and fast response times, use $\beta$ rays from ${ }^{63} \mathrm{Ni}$-foils or UV radiation from UV lamps as ionisation sources. Because of the limited selectivity of such IM spectrometers, more specific ionisation methods are highly desirable. The REMPI technique offers such a possibility. ${ }^{5-7}$ Tunable lasers, such as dye lasers, allow selective ionisation of compounds through variation of the excitation wavelength, thus probing the gas phase absorption spectrum of the respective analytes. In a one-colour two-photon experiment the first photon serves to occupy an excited state, from where the second photon ionises the molecule. Through this resonant two-photon excitation, the ionisation efficiency can be varied over several orders of magnitude. Consequently, analytes with similar ion mobilities, but differences in the absorption spectra, can be detected both qualitatively and quantitatively side by side. Another advantage of using lasers as an ionisation source in IM spectrometry is the possibility of investigating compounds with lower vapour pressures through the technique of laser desorption. ${ }^{7-9}$

At constant temperature and pressure, the ion mobilities of gas phase ions depend on the interaction potentials and the geometry of the colliding partners (molecular ion and drift gas). ${ }^{1}$ Consequently, IM spectrometry is a technique for the experimental determination of the structure of gas phase ions. The method, in connection with mass spectrometry, has already successfully been applied for the investigation of the formation and structure of C-clusters ${ }^{10,11}$ as well as for the elucidation of the structure of proteins, ${ }^{12}$ semiconductors and metal cluster ions. ${ }^{13-15}$ Combined with quantum chemical calculation methods, providing 
structure proposals, and with improved models for the calculation of diffusion cross sections, ${ }^{10,16}$ a powerful tool for structure determination of gas phase ions has been created. ${ }^{17}$

In this work, REMPI and IM spectra of several PAH and alkylbenzenes are presented. The experimentally determined diffusion cross sections $\left(\Omega_{\text {exp }}\right)$, directly obtained from ion mobilities (cf. equation 1), are compared with diffusion cross sections calculated on the basis of the EHSSM $\left(\Omega_{\text {calc }}\right)$. Such $\Omega_{\text {exp }} / \Omega_{\text {calc }}$-correlations are investigated for rigid molecules, like $\mathrm{PAH}$, and for flexible compounds, at the example of the hexylbenzene isomers. Taking the substance classes of alkylbenzenes and alkylphenylethers as an example, the potential of IM spectrometry in combination with REMPI for the selective detection of analytes in compound mixtures is demonstrated. In a first step, the REMPI spectra for several compounds were recorded. Then the spectral information was used to determine all molecules in a mixture of eight compounds. A third point of interest was the separation of alkylbenzene isomer mixtures. For such mixtures the wavelength-dependent selective detection of the individual isomers has been investigated.

\section{EXPERIMENTAL SECTION}

A home-built IM spectrometer, similar to that described by Gormally et al., ${ }^{18}$ was used in two different set-ups for volatile (gas phase experiment) and less volatile compounds (desorption experiment). The drift tube (drift length $d=10.5 \mathrm{~cm}$, inner diameter $3 \mathrm{~cm}$, electrical field $E=298.5 \mathrm{~V} \mathrm{~cm}^{-1}$ ) consisted of alternating rings made of Teflon and stainless steel. A more detailed description of the IM spectrometer was previously published. ${ }^{7}$ A laser system consisting of a Nd:YAG pump laser (Spectra Physics, Darmstadt, Germany, PRO 190-10 with second, third and fourth harmonic generation, laser pulse width $\tau_{\mathrm{L}} \approx 2-3 \mathrm{~ns}$, maximal laser pulse energy $E_{\mathrm{L}}=$ $275 \mathrm{~mJ}$ at $355 \mathrm{~nm}$, beam diameter $d_{\mathrm{L}}<10 \mathrm{~mm}$ and repetition rate $f=10 \mathrm{~Hz}$ ) pumping a tunable dye laser (Sirah, Kaarst, Germany, PRSC-LG-30) was used as REMPI excitation source. The dye laser was equipped with a resonator/preamplifier cell, a rectangular amplifier cell and a frequency conversion unit (FCU) for frequency doubling (SHG, efficiency around $20 \%$ ), the tuning range was 350 to $610 \mathrm{~nm}$, the line width 1.5 $\mathrm{pm}$ and the beam diameter after passing the FCU $d_{\mathrm{L}}<5 \mathrm{~mm}$. As laser dyes, coumarins 307 and 153 as well as rhodamines $6 \mathrm{G}$ and B (all from Sirah) dissolved in ethanol were used. Pumping at $355 \mathrm{~nm}$ yielded maximum efficiencies of around $15 \%$. The laser beam entered the IM spectrometer through a pinhole with 1 $\mathrm{mm}$ diameter and a quartz glass window. The laser energy at the location of the ionisation region was typically in the range 0.01 - $0.1 \mathrm{~mJ}$. Helium 5.0 (Messer Griesheim GmbH, Krefeld, Germany, $99.999 \%$ purity) was used as drift gas in order to obtain ion mobilities, which can be correlated with $\Omega_{\text {calc }}$ values obtained from the EHSSM. The volatile compounds, which were placed in diffusion tubes or permeation tubes, were introduced into the ionisation region by helium acting as carrier gas (sample gas). Concentrations were adjusted by appropriate dilution of the sample gas through mixing with a system of mass flow controllers (Umwelttechnik MCZ GmbH, Ober-Mörlen, Germany, MK 10) and by variation of the temperature by placing the sample tubes in an oven or cooling bath. Gas flow rates were typically $400 \mathrm{ml}$ $\mathrm{min}^{-1}$ for the drift gas and $150 \mathrm{ml} \mathrm{min}^{-1}$ for the sample gas. The less volatile compounds were dissolved in $\mathrm{n}$ pentane (Roth, Karlsruhe, Germany) or tetrahydrofuran (Sigma-Aldrich, Seelze, Germany) and around $50 \mu l$ of the solution were placed on an aluminium sample holder. After evaporation of the solvent the sample holder was introduced into the IM spectrometer. Desorption and ionisation of the sample was performed in a single step.

The determination of $\Omega_{\text {calc }}$ was carried out in two steps. The first step included a systematic conformation analysis followed by geometry optimisation of the individual conformations by semiempirical methods (PM 3). In the next step, the diffusion cross sections for the EHSSM were calculated with the program MOBCAL, developed by Shvartsburg and Jarrold. ${ }^{16}$ The results of the program MOBCAL are based on the following radii of the individual atoms: $R[C]=1.6 \AA, R[H]=1.2 \AA, R[O]=1.55 \AA$.

\section{RESULTS AND DISCUSSION}

\subsection{REMPI spectra and selective mixture analysis}

Besides an increase in sensitivity, the advantage of using lasers as ionisation source in IM spectrometry is the introduction of "optical selectivity". Thus, REMPI-based IM spectrometry is a combination of two analytical tools, namely gas phase absorption spectroscopy and IM spectrometry, and probes two different parameters, namely absorption coefficient and ion mobility. There are only few publications, which report on REMPI 
spectra at atmospheric pressure. Furthermore, most REMPI spectra were recorded in ionisation cells, which cannot resolve the formed ions. In such cells it is not possible to distinguish between parent and fragment ions and ions formed by impurities. REMPI spectra of nine compounds (benzene, toluene, anisole, o-, pxylene, o-, p-methylanisole and o-, p-dimethoxybenzene), measured in our IM spectrometer at room temperature, are displayed in Fig.1.
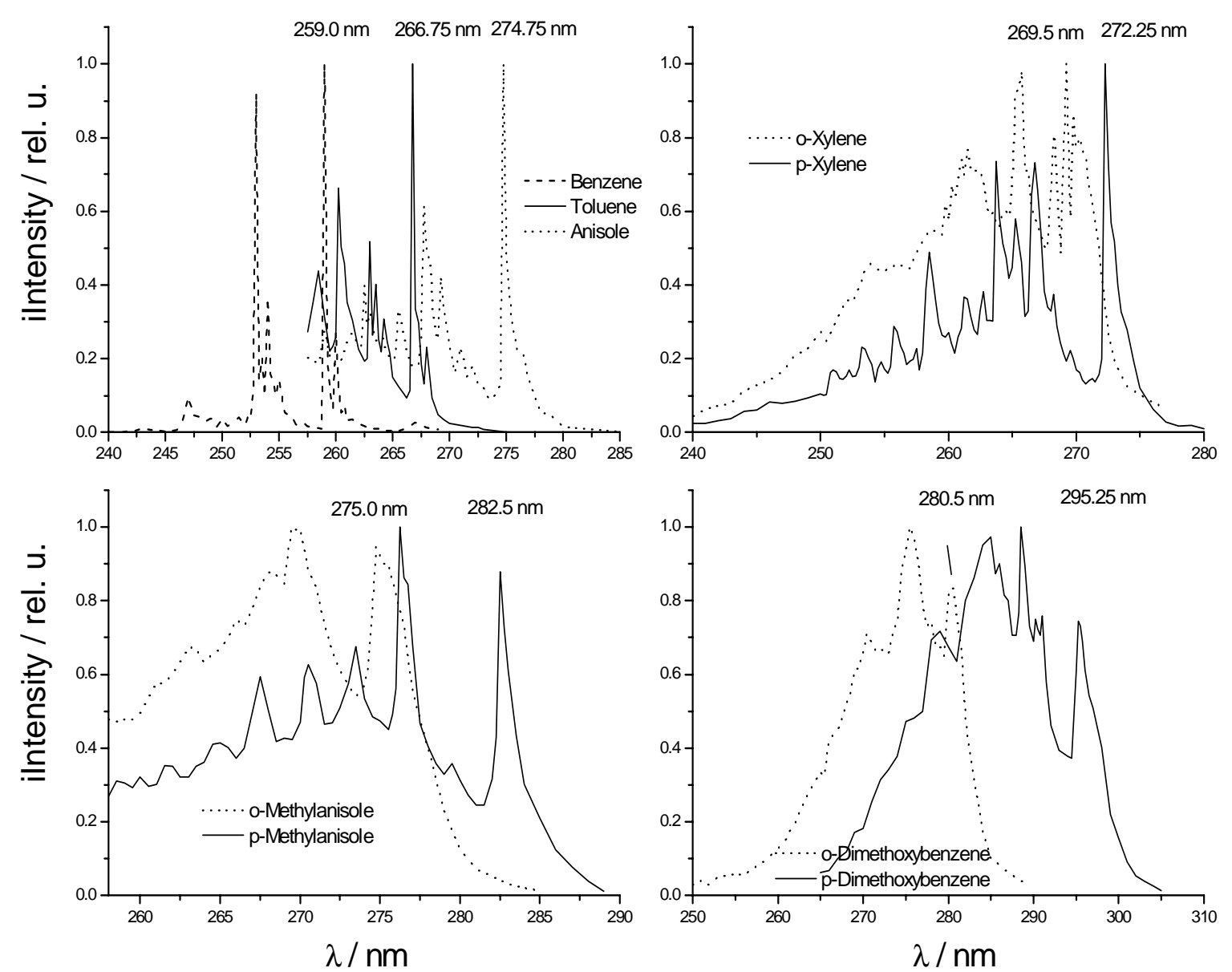

Fig. 1. REMPI spectra of benzene, toluene, anisole, $o$ - and $p$-xylene, $o-$ and $p$-methylanisole, $o$ - and $p$ dimethoxybenzene.

At higher temperature the spectra lose resolution. ${ }^{19}$ The laser energy of less than $100 \mu \mathrm{J}$ did not lead to the formation of fragment ions. The REMPI spectra resemble the UV absorption spectra (not shown). Especially the REMPI spectra of benzene, toluene and anisole show a fine structure, which should enable a selective detection of these three compounds in mixtures. The spectra of the 0 - and p-isomers of xylene, methylanisole and dimethoxybenzene indicate the possibility to distinguish between the 0 - and $p$-isomers by the red spectral shifts in their REMPI spectra. The expected and observed red spectral shifts with the introduction of ring substituents increase from methyl to methoxy group, and with enhancing number of substituted groups. That is shown in Fig. 1 with orderings of red spectral shift for benzene $<$ toluene $<$ anisole, for o-xylene < o-methylanisole < o-dimethoxybenzene, and for $\mathrm{p}$-xylene $<\mathrm{p}$-methylanisole $<\mathrm{p}$ dimethoxybenzene.

The significant increase of sensitivity and selectivity of REMPI-based IM spectrometry is shown with the example of a complex mixture of 8 alkylbenzenes and alkylphenylethers containing toluene, anisole, and the ortho- and para-substituted isomers of xylene, methylanisole and dimethoxybenzene. Despite the small differences in the structures of the eight compounds, the IM spectra show five almost baseline-resolved 
peaks (Fig.2, I-V), which belong to substances with different molecular weights. These are toluene, anisole and the isomers of xylene, methylanisole and dimethoxybenzene. This is in agreement with the $\Omega_{\text {calc }}$ values which show larger differences between these compounds and only small differences between the ortho- und para-isomers, whose mobility differences are within the resolution limit of our IM spectrometer. The orthoand para-isomers can be distinguished by their spectrally shifted REMPI spectra. The para-isomers are detected alone at their absorption maximum, while at the peak maximum of the ortho-isomer both compounds are ionized. Therefore, the qualitative and quantitative determination of all analytes in a combination of spatial (separation in the drift tube) and spectral resolution (appropriate ionisation wavelength) is demonstrated. Consequently, analytes with similar ion mobilities but different absorption spectra can be distinguished. In Fig.2, strong changes in the IM spectra dependent on the two-photon excitation wavelengths are notable. Here another advantage of REMPI becomes evident. If in a mixture one substance is available only at low concentrations, high ionisation efficiency for the trace compound, and discrimination against the major components can often be obtained by selecting the appropriate REMPI wavelengths. Large background signals, potentially inundating IM spectra of interest and possibly causing reduced resolution due to electrostatic repulsion, can thus be avoided.

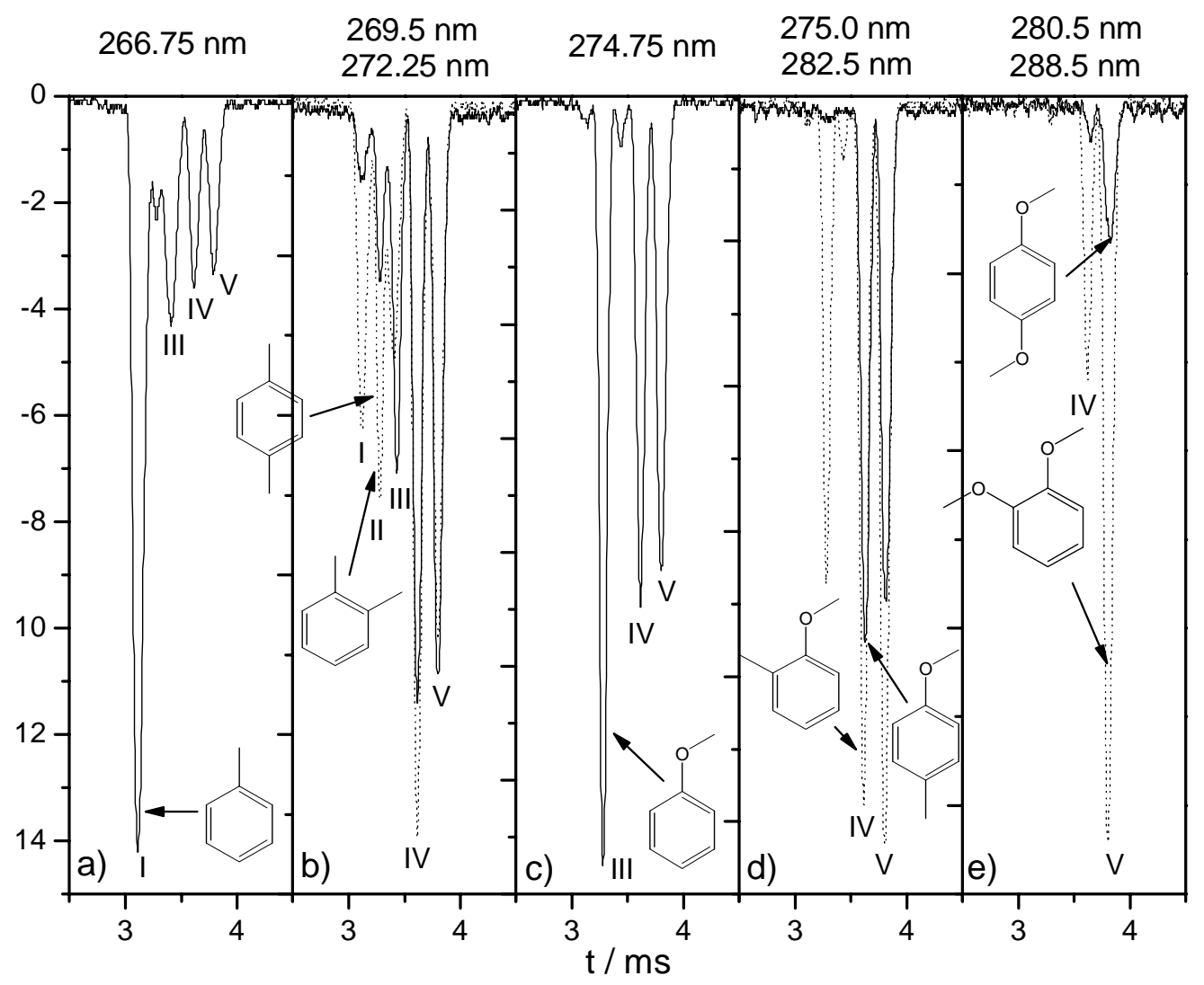

Fig. 2. REMPI-based IM spectra of an eight-component mixture obtained with different two-photon excitation wavelengths.

In IM spectrometry at ambient pressure and room temperature, multiple ion-molecule collisions take place. Dependent on ionisation potentials and electron affinities of the partners, the conditions for electron transfer (ET) reactions are thus given. ${ }^{20}$ Therefore, ET reactions for some substance pairs (toluene-anisole, toluenep-xylene, toluene-methylanisole, toluene-dimethoxybenzene, anisole-dimethoxybenzene) were investigated, applying the same experimental conditions as in the substance mixture. With the exception of mixtures of toluene and p-xylene, no indication for ET reactions could be observed. The experiments show that ET reactions can be important and have to be regarded in analytical applications, especially in the case of a 
concentration excess of one compound in a mixture. Under our experimental conditions concentration discrimination by ET reactions can be neglected.

In comparison to molecular beam spectra, the resolution of REMPI spectra in IM spectrometry is relatively low mainly because the molecules are in thermal equilibrium at atmospheric pressure and room temperature. However, the usefulness of REMPI-based IM spectrometry could be demonstrated in our example.

\subsection{Correlations of experimental and calculated diffusion cross sections}

The mobility of the ions in a homogeneous electric field (field strength $E$ ) and bath gas depends on the structure of both partners. The theory developed by Mason et al ${ }^{21}$ gives the following equation, showing an indirect proportionality between ion mobility and diffusion cross section:

$$
K=\frac{v_{D}}{E}=\frac{3 \sqrt{2 \pi}}{16} \cdot \frac{q}{N_{\text {Gas }}} \cdot \sqrt{\frac{1}{M_{\text {Ion }}}+\frac{1}{m_{\text {Gas }}}} \cdot \frac{1}{\sqrt{k_{B} T}} \cdot \frac{1}{\Omega}
$$

In this equation, $q$ is the ion charge, $N_{\text {Gas }}$ the bath gas density, $M_{\text {lon }}$ and $m_{\text {Gas }}$ are the masses of ion and bath gas molecules, $\mathrm{k}_{\mathrm{B}}$ is the Boltzmann constant, $T$ the temperature and $\Omega$ the diffusion cross section. $\Omega_{\exp }$ is obtained from the measured drift velocities $v_{\mathrm{D}}$, whereas $\Omega_{\text {calc }}$ is calculated as collision integral by averaging the momentum transfer cross section over the relative velocity and the collision geometry. To evaluate $\Omega$ for polyatomic ions in a rigorous treatment, using realistic potentials, requires extreme computational efforts. Using helium as drift gas with low polarisability, the interaction potentials between molecular ion and drift gas atoms can be neglected in most cases. The atoms in molecules and clusters can be approximated as hard spheres, and therefore the interactions are described as collisions between hard spheres. The projection model (PM) was introduced, which determined the shadow of the molecule averaged over all collision angles.

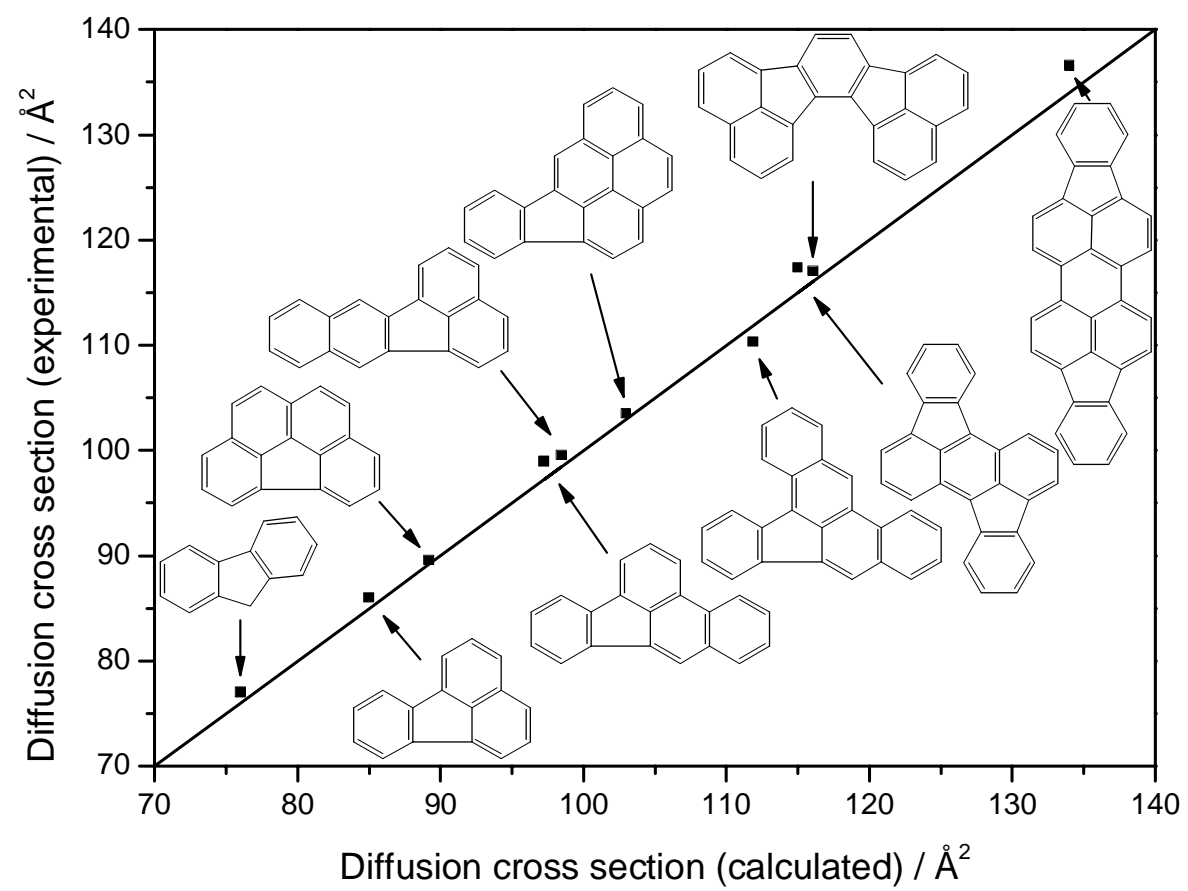

Fig. 3 Correlation of experimental and calculated diffusion cross sections of the fluoranthenes. The straight line with slope unity indicates the $1: 1$ identity of $\Omega_{\text {exp }}$ and $\Omega_{\text {calc }}$.

More ambitious models, like the exact hard sphere scattering model (EHSSM) introduced by Shvartsburg and Jarrold, ${ }^{16}$ treat the collision process more rigorous. The EHSSM takes into account multiple collisions at the concave regions of the molecule and shadows on molecule parts in the collision process. In the case of pure convex surfaces the collision integrals of PM and EHSSM are equal. In the case of concave regions, the 
results of PM and EHSSM can deviate up to $20 \%$. As a criterium for correct description of a structure, measured and calculated cross section should deviate less than $2 \%$.

The drift time spectra of PAH, alkylbenzenes and alkylphenylethers were recorded in gas phase (monocyclic molecules) and desorption experiments (PAH). Drift times were measured against anisole and naphthalene as standards, eliminating the daily fluctuations and reducing relative standard deviations below $1 \%$. The method used in this work is based on the comparison with standards of known ion mobility. ${ }^{22,23}$ Reasons for the application of this method are possible variations and inhomogeneities in the electric drift field and changes of the drift length caused by thermal effects.

The ion mobilities of benzene and biphenyl were measured and found to be in a good agreement with published data. ${ }^{14}$ In comparison to nitrogen or air, only a small number of ion mobility studies in helium can be found in the literature.

$\Omega_{\text {exp }} / \Omega_{\text {calc }}$-correlations allow the verification of model calculations and experimental technique. The $\Omega_{\text {calc }}$ calculations are easily performed with only one conformation. Examplary, the high quality of these correlations is shown for the compound classes of fluoranthenes (Fig.3) and [N]phenylenes (Fig.4). The comparison with the ideal 1:1 straight line underlines the excellent agreement between $\Omega_{\exp }$ and $\Omega_{\text {calc }}$. For both compound classes, the correlation between inverse ion mobilities and molecular weight is also very good (not shown). The fluoranthenes are flat and rigid molecules, whereas some structural flexibility can be expected for the [4]phenylenes. Most of these compounds are not investigated with IM spectrometry up to now, therefore the ion mobilities were measured here for the first time. For the fluoranthenes, the correlation includes 10 compounds starting from fluorene and fluoranthene to the larger benzofluoranthenes. For the [N]phenylenes, the correlation includes 7 compounds ranging from the biphenylene to the triangular [5]phenylene. It is remarkable that the [4]phenylenes with their different structures, namely angular [4]phenylene, triangular [4]phenylene and zig-zag [4]phenylene, show diffusion cross sections that are nearly identical.

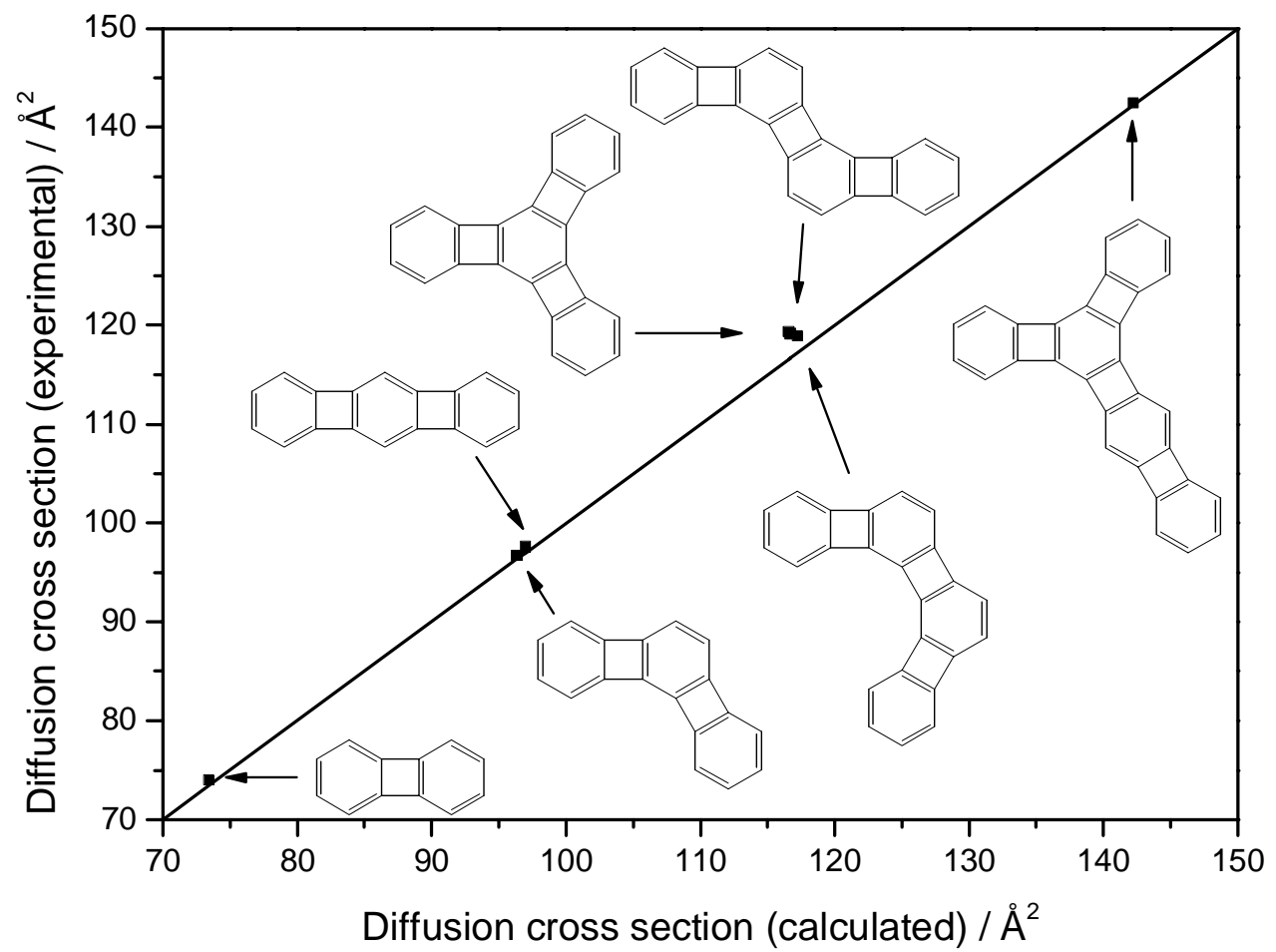

Fig. 4. Correlation of experimental and calculated diffusion cross sections of the [N]phenylenes, with $\mathrm{N}$ denoting the number of benzene units. The straight line with slope unity indicates the $1: 1$ identity of $\Omega_{\exp }$ and $\Omega_{\text {calc }}$.

This is also the case for the [3]phenylenes, namely angular [3]phenylene and linear [3]phenylene. Experimental evidence suggests a low-frequency vibrational motion in the [4]phenylenes, ${ }^{24}$ which may 
consequently not be completely planar. Such vibrations would probably increase the calculated $\Omega_{\text {calc }}$ values, as can perhaps be seen in the small deviation from the ideal 1:1 straight line. This explanantion, however, is tentative due to the limited resolution of the IM spectrometer.

\subsection{Possibilities and limits of the isomer separation}

One advantage of IM spectrometry is the potential for separation of isomers. This is based on the direct proportionality of drift time and diffusion cross section. The latter can be different for isomers with different molecular structures. The limits and possibilities of isomer separation were investigated systematically in regard to the experimental resolution available in IM spectrometry.

Other aspects of isomer investigation are the test of the ion mobility correlations and the "optical selectivity" for the ionisation. A suitable substance group for these investigations are the alkylbenzenes, with the isomers of hexylbenzenes as example. The investigations were extended to alkylphenylethers.

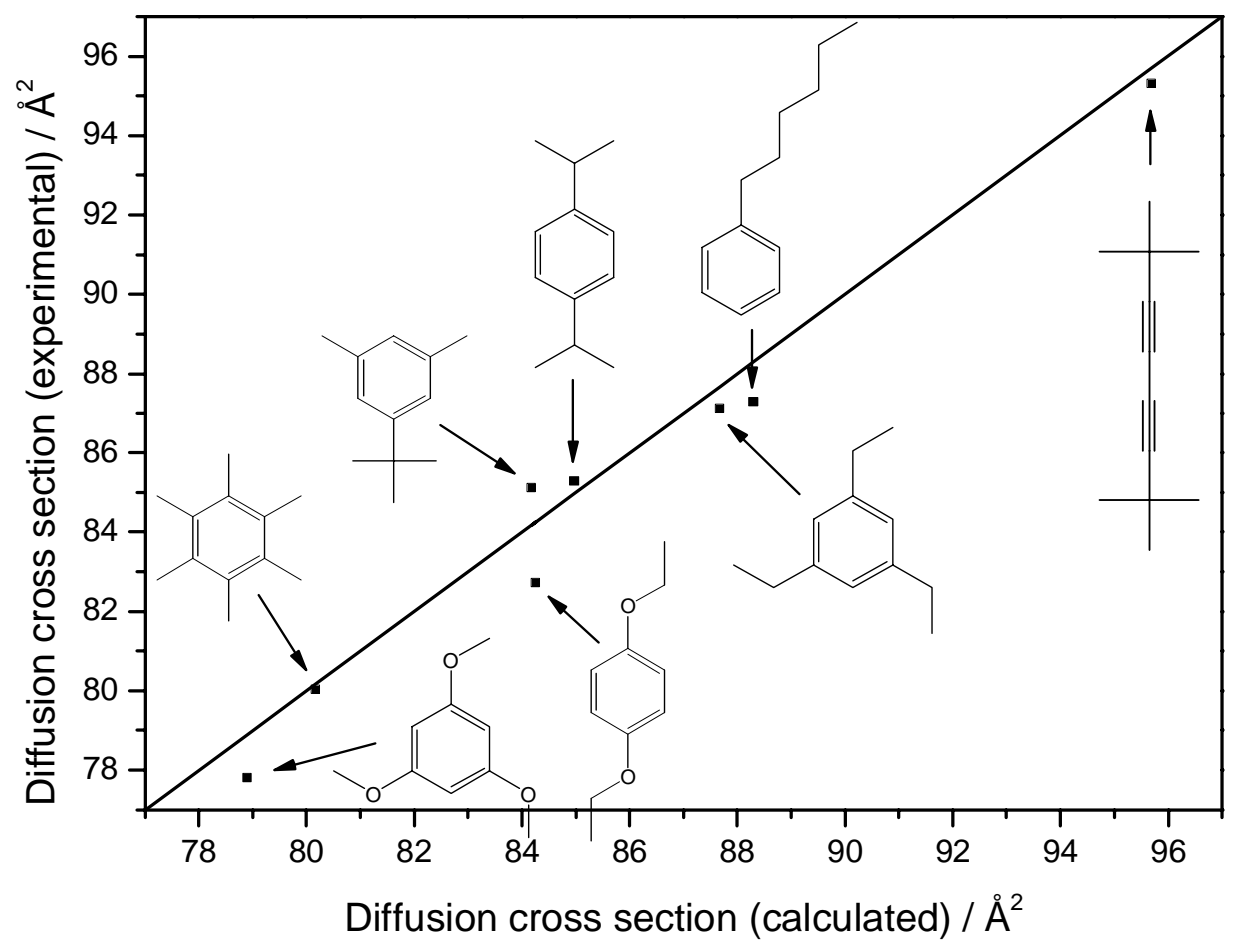

Fig. 5. Correlation of experimental and calculated diffusion cross sections of the hexylbenzenes. The straight line with slope unity indicates the $1: 1$ identity of $\Omega_{\exp }$ and $\Omega_{\text {calc }}$.

As a first step, using the measured ion mobilities, the $\Omega_{\text {exp }} / \Omega_{\text {calc }}$-correlation is considered. As is evident from Fig. 5 the correlation is excellent, with deviations from the ideal $1: 1$ straight line of less than $2 \%$. The more compact structures of the hexylbenzene isomers, like the hexamethylbenzene and 1.3.5-trimethoxybenzene, have about $9 \%$ lower diffusion cross sections than the more stretched structures, like the n-hexylbenzene and 1.3.5-triethylbenzene. If we include 2.2.7.7-tetramethyl-3.5-octadiyne, the overall variation in $\Omega$ increases to about $17 \%$. The isomeric compounds under investigation are hence clearly distinguishable with IM spectrometry. The large difference of nearly $10 \%$ in the diffusion cross sections between 1.3.5triethylbenzene and 1.3.5-trimethoxybenzene, based on the exchange of the methylene group by the oxygen atom, is also remarkable. In a second step, REMPI spectra obtained from mixtures of three isomers are considered (Fig.6). For the hexylbenzenes, the distinction of three isomers in a mixture is possible. While the peak of hexamethylbenzene and the peak pair of $n$-hexylbenzene and $p$-isopropylbenzene are baseline separated, for the determination of the single components of the peak pair differences in the UV spectra have to be used. The red shift of the UV spectrum of $p$-isopropylbenzene can be used to distinguish between both 
compounds. Therefore, the ionisation at different wavelengths allows a selective determination of all components in the isomer mixture, what is an impressing example for selectivity in IM spectrometry.

In summary it can be concluded that for the distinction of isomers in mixtures, IM spectrometry is a suitable tool. Its applicability was demonstrated for hexylbenzene isomers. Limits of spectral and spatial resolution also became obvious. This was more relevant when we investigated the isomer separation of alkylbenzenes with shorter alkyl chains, which is not shown here. It is obvious that the distinction of isomers increasingly succeeds, if the variable structural part (i.e., the alkyl side chains) becomes more spacious compared to the constant structural part (i.e., the phenyl ring), as it is the case for the isomers of the higher homologues of alkylbenzenes.

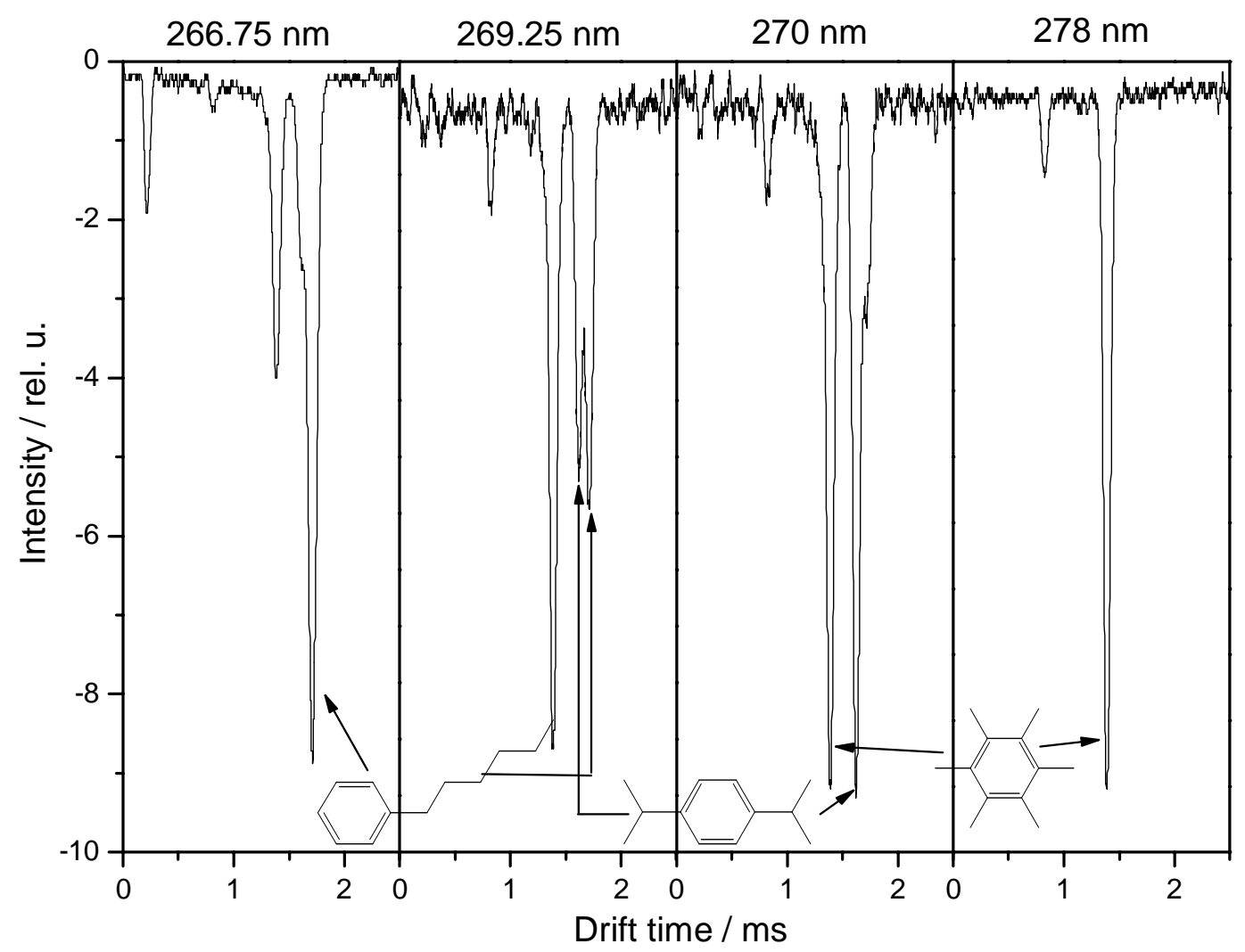

Fig. 6. REMPI-based IM spectra of a mixture of three isomers of hexylbenzene.

\section{CONCLUSION}

The correlations of the measured and calculated diffusion cross sections for the rigid PAH and alkylbenzenes show that the theoretical calculations on the basis of the EHSSM present an easy and good description for ion-molecule interactions in helium as drift gas and indicate the reliability of the model and method. The limits of the method became obvious with the correlation of the isomers, which show only small differences in ion mobilities.

The REMPI spectra of nine alkylbenzenes and alkylphenylethers are investigated. On the basis of these spectra, the qualitative distinction of eight compounds in a mixture is shown. The experiments on the selectivity of ionisation of the alkylbenzene and alkylphenylether mixtures by REMPI show that the combination of two analytical tools, namely gas phase absorption spectroscopy and IM spectrometry, that is the combination of spectral resolution and spatial separation, presents a promising sensing method. The most remarkable property of REMPI-based IM spectrometry is its usefulness for isomer separation. 


\section{Acknowledgement}

This work was financially supported by the German Ministry of Education and Research (BMBF) in the framework of the MILAN project (FKZ: 13N8099).

\section{References}

1. G.A. Eiceman and Z. Karpas, Ion Mobility Spectrometry, CRC Press, Boca Raton, 1992.

2. J.I. Baumbach and G.A. Eiceman, Applied Spectroscopy, 1999, 53, 338A.

3. K. Cottingham, Anal. Chem., 2003, 75, 435A.

4. H. Borsdorf, M. Rudolph, Int. J. Mass Spectr., 2001, 208, 67.

5. C. Illenseer, H.-G. Löhmannsröben, R.H. Schultze, J. Environ. Monit., 2003, 5, 780.

6. D.M. Lubman and M.N. Kronick, Anal. Chem., 1982, 54, 1546.

7. C. Illenseer and H.-G. Löhmannsröben, Phys. Chem. Chem. Phys., 2001, 3, 2388.

8. G.A. Eiceman, G.K. Anderson, W.C. Danen, M.J. Ferris and J.J. Tiee, Anal. Lett., 1988, 21, 539.

9. D. Young, K.M. Douglas, G.A. Eiceman, D.A. Lake and M.V. Johnston, Anal. Chim. Acta, 2002, 453, 231.

10. G. von Helden, M.-T. Hsu, P.R. Kemper and M.T. Bowers, J. Phys. Chem., 1991, 95, 3835.

11. G. von Helden, M.-T. Hsu, N. Gotts and M.T. Bowers, J. Phys. Chem., 1993, 97, 8182.

12. D.E.Clemmer and M.F. Jarrold, J. Mass Spectrom. 1997, 32, 577.

13. M. Jarrold and J. Bower, J. Phys. Chem., 1993, 97, 1746.

14. A. Shvartsburg and M. Jarrold, Chem. Phys. Lett., 2000, 317, 615.

15. S. Gilb, P. Weis, F. Furche, R. Ahlrichs and M.M. Kappes, J. Chem. Phys., 2002, 116, 4094.

16. A. A. Shvartsburg and M. F. Jarrold, Chem. Phys. Lett., 1996, 261, 86-91.

17. M. Krishnamurthy, J.A. de Gouw, V.M. Bierbaum and S.R. Leone, J. Phys. Chem., 1996, 100, 14908.

18. J. Phillips and J. Gormally, Int. J. Mass Spectrom. Ion Processes, 1992, 112, 205.

19. D.M. Lubman and M.N. Kronick, Anal. Chem., 1983, 55, 1486.

20. G.A. Eiceman and V.J. Vandiver, Anal. Chem., 1986, 58, 2331.

21. E.A. Mason and E.W. McDaniel, Transport Properties of lons in Gases, Wiley, New York, 1988.

22. G.A. Eiceman, E.G. Nazarov, J.A. Stone, Anal. Chim. Acta, 2003, 493, 185.

23. C.S. Creaser, M. Benyezzar, J.R. Griffiths, J.W. Stygall, Anal. Chem., 2000, 72, 2724.

24. C. Dosche, M.U. Kumke, F. Ariese, A.N. Bader, C. Gooijer, P.I. Dosa, S. Han, O.S. Miljanic, K.P.C. Vollhardt, R. Puchta, N.J.R. van Eikema Hommes, Phys. Chem. Chem. Phys., 2003, 5, 4563. 